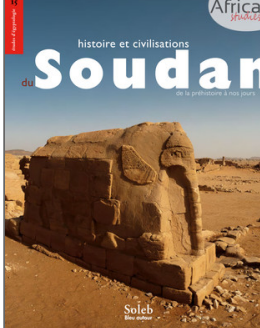

Histoire et civilisation du Soudan

De la préhistoire à nos jours

\title{
7. Le Soudan du Sud, cinq ans après l'indépendance
}

\section{Bernard François}

DOI : 10.4000/books.africae. 2917

Éditeur : Africae, Soleb, Bleu autour

Lieu d'édition : Paris, Khartoum

Année d'édition : 2017

Date de mise en ligne : 17 janvier 2022

Collection : Africae Studies

EAN électronique : 9782493207074

\section{(2) OpenEdition}

\section{Books}

http://books.openedition.org

\section{Référence électronique}

FRANÇOIS, Bernard. 7. Le Soudan du Sud, cinq ans après l'indépendance In : Histoire et civilisation du Soudan: De la préhistoire à nos jours [en ligne]. Paris, Khartoum : Africae, 2017 (généré le 28 janvier 2022). Disponible sur Internet : <http://books.openedition.org/africae/2917>. ISBN : 9782493207074 DOI : https://doi.org/10.4000/books.africae.2917. 


\section{le Soudan du Sud, cinq ans après l'indépendance}


C'est à la quasi-unanimité ( $98,83 \%$ des voix) que les Soudanais du Sud se sont prononcés en faveur de l'indépendance lors du référendum organisé dans la semaine du 9 au 15 janvier 2011. Mais avant même l'indépendance, célébrée dans l'euphorie le 9 juillet 2011, le Soudan du Sud,

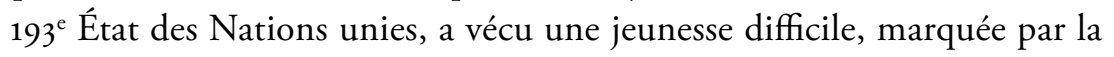
résurgence du conflit avec le régime de Khartoum - qui trouvera une solution négociée - , puis par de violentes luttes internes qui se poursuivaient encore à l'été 2016.

\section{Le conflit entre le Soudan et le Soudan du Sud mai 20||-avril 20|3}

Durant les cinq années de la trêve obtenue par l'accord de paix global (Comprehensive Peace Agreement - CРA) signé cinq ans plus tôt, aucun accord n'est intervenu dans les négociations sur le tracé exact de la frontière, (en particulier à propos de la région d'Abyei), ni sur la répartition des revenus tirés des ressources pétrolières (le Soudan du Sud possède maintenant les trois quarts des ressources pétrolières de l'ex-Soudan unitaire).

Le 21 mai 2011, l'armée nordiste s'attaque à la région d'Abyei. Elle recourt à des chars d'assaut, ce qui force les rebelles de l'Armée populaire de libération du Soudan (ApLs) à battre en retraite. Elle fait aussi intervenir l'aviation, qui bombarde plusieurs villages de la région. Le Nord présente son offensive comme une réaction à l'attaque menée le 19 mai par l'Apls, qui la nie, contre un convoi de troupes nordistes et de soldats du maintien de la paix de l'ONU.

Le 22, Khartoum annonce la prise de la région d'Abyei et, par la voix du ministre d'État Amin Hassan Omar, affiche sa volonté de la nettoyer des "groupes armés du Sud». Le Conseil de sécurité des Nations unies appelle Khartoum, qui s'y refuse, à retirer ses troupes de cette région contestée. Des milliers de civils fuient les combats et, selon l'ONU, des cas de pillages et d'incendies se produisent dans la région. La secrétaire d'État américaine Hillary Clinton demande au Nord de retirer ses troupes de la région d'Abyei et dit soutenir la proposition de l'Éthiopie.

Le 23, le Soudan du Sud réagit en accusant le Nord de provoquer une nouvelle guerre civile. Le lendemain, le président soudanais Omar el-Bechir affirme dans un discours à Khartoum qu' "Abyei appartient au Nord-Soudan".

Le 25, le secrétaire général de l'ONu, Ban Ki-moon, propose la mise en place d'une nouvelle force de maintien de la paix au Soudan, forte de 7000 hommes, alors que dans la même journée quatre hélicoptères de l'ONU ont été la cible de tirs dans la région d'Abyei, probablement 
le Soudan

\section{6}

de 1820

à nos jours de la part des forces nordistes. Le lendemain, Salva Kiir, le leader du Soudan du Sud affirme qu'il n'y aura pas de nouvelle guerre pour le contrôle de cette région contestée.

Le 28 mai, l'armée nord-soudanaise déclare mettre fin à ses opérations militaires dans la région. Le 31, l'Union africaine annonce que les deux camps acceptent d'établir une zone démilitarisée et l'Éthiopie se dit prête, si nécessaire et si les deux camps le souhaitent, à envoyer des troupes de maintien de la paix.

Le 5 juin 2011, les combats reprennent, cette fois dans le Nord, au Kordofan du Sud ${ }^{1}$, faisant plusieurs tués et provoquant la fuite de la population civile. Le 8 , le Soudan du Sud appelle à un cessez-le-feu, tout en accusant le Nord d'avoir bombardé un village dans l'État d'Unité le 10 juin. L'ApLs se dit prête à faire face à une offensive terrestre du Nord.

Le 11, Khartoum accepte des pourparlers de paix avec le Sud concernant la région d'Abyei, alors qu'aucun cessez-le-feu n'est toujours prévu et que les combats continuent de faire rage, notamment dans le Sud Kordofan.

Le 12, Khartoum, par la voix du président Omar el-Bechir, accepte de retirer ses troupes de la région contestée avant le 9 juillet, date prévue de l'indépendance du Soudan du Sud. Le lendemain, l'ApLs accuse le Soudan d'avoir mené un nouveau bombardement sur son territoire par l'emploi de MiG-23 et d'Antonov. Les deux camps acceptent par ailleurs dans la même journée qu'une zone démilitarisée soit créée dans la région d'Abyei et qu'y soient envoyés, sous l'égide de l'Union africaine, des soldats éthiopiens de maintien de la paix.

1 Outre Abyei, le contrôle du pétrole et la délimitation de la frontière, un autre important sujet de friction entre les deux Soudans est le statut de deux autres régions frontalières que sont les États du SudKordofan et du Nil Bleu. Bien que ces deux États appartiennent officiellement au Soudan, des mouvements d'opposition et des mouvements rebelles armés s'y opposent à la souveraineté de celui-ci; leurs leaders sont les anciens représentants du Spla au Nord: ces mouvements entretiennent donc des liens forts avec le Spla du Soudan du Sud. Le conflit tient notamment à la concentration du pouvoir et des ressources par le centre du Soudan, au détriment de sa périphérie à laquelle ces deux régions appartiennent. Ces problèmes latents avaient été ignorés dans le cadre de l'accord de paix global de 2005 (qui avait pris en compte, à l'inverse, les aspirations à l'indépendance de l'actuel Soudan du Sud). 
Le 14 juin 2011, des miliciens sudistes locaux passent à l'action; selon un porte-parole de l'ApLs, ils tuent sept policiers soudanais et vingt-deux civils près de la région d'Abyei. Le président des États-Unis, Barack Obama, appelle les deux camps à un cessez-le-feu, soulignant "qu'il n'y a pas de solution militaire» et accusant le Nord d'avoir provoqué le conflit.

Le 19, alors que les combats s'intensifient au Sud Kordofan, Khartoum y envoie des renforts blindés. L'armée soudanaise attaque également au Darfour les rebelles de l'Armée de libération du Soudan (ALs).

Le 20, les deux camps acceptent la démilitarisation de la zone contestée d'Abyei et l'envoi de soldats éthiopiens de maintien de la paix sous l'égide de l'ONU, mettant ainsi fin au conflit.

Le 9 juillet le Soudan du Sud devient officiellement indépendant. Le 14, l'Assemblée générale des Nations unies vote par acclamation l'admission de la République du Soudan du Sud, qui devient le $193^{\mathrm{e}}$ membre de l'ONU.

Les combats continuent en septembre, malgré la signature d'un cessez-le-feu.

Les 8 et 9 octobre a lieu la première visite à Khartoum de Salva Kiir en tant que président du Soudan du Sud. Les deux pays fixent un calendrier pour régler leurs différends persistants.

Le 10 novembre, des Antonov de l'armée de l'air soudanaise bombardent un camp dans l'État d'Unité, faisant 12 morts et blessés.

Le 12 décembre, les deux Soudans s'affrontent à Jau, un village du Soudan du Sud situé près de la frontière commune des deux États et dont l'appartenance est disputée.

Le 26 février 2012, les rebelles du Front révolutionnaire soudanais (FRs) affirment avoir tué jusqu'à 130 soldats dans une attaque contre une garnison de l'armée soudanaise à la frontière avec le Soudan du Sud.

Le $1^{\text {er }}$ mars, le Soudan du Sud accuse le Nord d'avoir bombardé deux puits de pétrole, ce que Khartoum conteste officiellement.

Le 26, les Nord-Soudanais bombardent l'État d'Unité au Soudan du Sud et déploient des troupes à la frontière. Les Sud-Soudanais contreattaquent et s'emparent des champs pétroliferes de Heglig, qui fournissent la moitié de la production pétrolière du Nord.

Le 30 , de nouveaux combats sont signalés près de la frontière entre l'armée soudanaise et les rebelles pro sud-soudanais.

Le 15 avril, des avions nord-soudanais bombardent le village frontalier de Heglig, conquis par les Sud-Soudanais une semaine auparavant.

Le 22, le Soudan du Sud déclare s'être complètement retiré de la zone de Heglig, dont les installations ont été sérieusement endommagées par les combats. L'armée soudanaise affirme que l'ApLs a perdu 1200 hommes. 
Dans la nuit du 23 au 24 avril 2012, le Nord procède, jusqu'à $25 \mathrm{~km}$ au sud de la frontière, à de nouveaux bombardements aériens qui font 16 morts et 34 blessés.

Le 27 septembre sont signés par les belligérants des accords de sécurité et de coopération qui portent notamment sur la mise en place d'une zone tampon démilitarisée.

Après plusieurs mois de tensions, les deux gouvernements réussissent à trouver un modus vivendi sur le montant de la redevance que le Soudan du Sud, pays enclavé, doit payer à Khartoum pour pouvoir utiliser ses oléoducs. C'est en avril 2013, après plus d'un an d'interruption, que le pétrole sud-soudanais transite à nouveau par le Soudan. Les deux États mettent également en œuvre les accords de sécurité et de coopération; la zone tampon prévue voit ainsi le jour.

\section{Luttes de pouvoir et guerre civile depuis juillet 2013}

le Soudan

\section{8}

de 1820

à nos jours

\section{La guerre civile}

— Juillet 2013: le Mouvement populaire de libération du Soudan (MpLs), qui gouverne le Soudan du Sud depuis son indépendance, est secoué par une lutte acharnée pour le pouvoir. Elle oppose le président, Salva Kiir, et son vice-président, Riek Machar, issus des deux principaux groupes ethniques du pays, les Dinka pour Salva Kiir et les Nuer pour Riek Machar. Salva Kiir limoge l'ensemble de son gouvernement, ainsi que son vice-président.

- Décembre 2013: la lutte politique se transforme en guerre civile lorsque le président annonce avoir déjoué un coup d'État fomenté contre lui par son ancien vice-président. Ce dernier nie pour sa part toute tentative de putsch, avant d'appeler l'armée à renverser Salva Kiir, que Riek Machar accuse de son côté de vouloir "allumer une guerre ethnique». Dès le 15 décembre, d'intenses combats opposent des factions rivales au sein de l'armée. Ils s'étendent à plusieurs régions du pays et s'accompagnent d'atrocités ethniques (tueries, pillages et viols). Riek Machar réussit à quitter Juba pour regagner son fief dans le nord, d'où il mène ses représailles contre l'armée gouvernementale et ses alliés.

- 2014-2015: la guerre civile sud-soudanaise, qui durera vingt mois, touche plus particulièrement les villes du nord et du nord-est, épargnant le sud et l'ouest du pays. Les villes de Bentiu (nord), Bor (est) et Malakal (nord-est) sont les principaux foyers du conflit.

S'agit-il véritablement d'une guerre ethnique? Pour nombre de chercheurs, il s'agit d'abord d'une lutte pour le pouvoir, non d'une guerre entre les Dinkas et les Nuer. En effet, de nombreuses ethnies (Bari, 


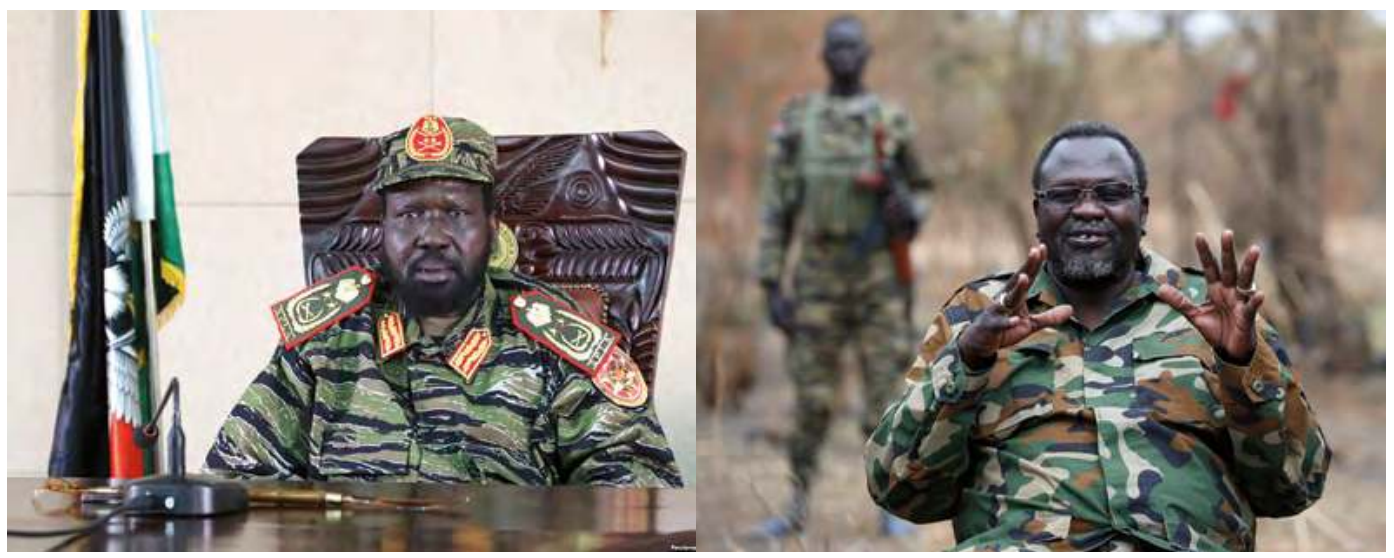

Salva Kiir, Mpls, président du Soudan du Sud.

Riek Machar, MpLs, vice-président du Soudan du Sud.

Zandé...) entrent en jeu au-delà de ces deux grands groupes, et les allégeances sont changeantes entre les combattants qui se livrent bataille parfois entre eux, à l'intérieur même de leurs clans respectifs.

Toujours est-il que la guerre civile sud-soudanaise fait plus de 50000 morts et plus de 2,5 millions de déplacés. En 2015, selon les rapports des agences des Nations unies et des $\mathrm{O}_{\mathrm{NG}}$, quelque cinq millions de personnes — soit plus d'un tiers de la population — ont eu besoin d'aide humanitaire pour survivre.

- Janvier 2014: des combats opposent l'armée sud-soudanaise aux rebelles menés par l'ex-vice-président Riek Machar dans la ville pétrolière de Malakal; le 23 janvier 2014, un accord de cessez-le-feu est signé.

- 12 mars: l'Union africaine crée une commission d'enquête chargée de déterminer les responsables des atrocités commises depuis l'ouverture, à la mi-décembre, du conflit entre les forces gouvernementales et les troupes loyales à l'ancien vice-président Riek Machar.

- 17 avril: environ 350 jeunes hommes, armés et en civil, attaquent l'enceinte de l'ONu à Bor, où près de 5000 civils, essentiellement Nuer, ont trouvé refuge; bilan: 58 morts.

— 21 avril: l'ONU accuse les troupes de Riek Machar d'avoir massacré des centaines de civils sur des bases ethniques lorsqu' elles ont repris la ville de Bentiu.

— 10 juin : le président Salva Kiir et le chef de la rébellion, Riek Machar, s'accordent sur la formation d'un gouvernement de transition sous soixante jours.

- 25 juillet: dans une déclaration unanime, les quinze pays membres du Conseil de sécurité de l'ONU «se déclarent très inquiets de la situation d'insécurité alimentaire catastrophique au Soudan du Sud, qui est désormais la pire au monde». 
- 15 août: malgré les menaces de sanctions de l'ONU, d'intenses combats reprennent dans le pays, opposant des partisans de l'ancien vice-président Riek Machar à ceux du président Salva Kiir.

- 22 septembre: des négociations débutent en Éthiopie sous l'égide de l'Autorité intergouvernementale pour le développement (Igad), organisation régionale est-africaine; elles visent à trouver une issue durable au conflit mais seront suspendues le 5 octobre en attente d'un accord.

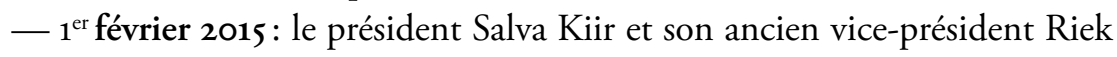
Machar scellent un accord de cessez-le-feu, le... septième en un an.

- 20 mars: un rapport de l'Unicef estime qu'environ 12000 enfants, essentiellement des garçons, ont été enrôlés comme soldats, aussi bien par le gouvernement que par les groupes rebelles, dans cette guerre civile.

-8 mai 2015: les combats qui font rage dans le nord provoquent un exode massif de la population: selon l'ONU, près de cent mille déplacés en une semaine. Ces affrontements sont parmi les plus meurtriers depuis qu'a débuté le conflit, en décembre 2013: plusieurs dizaines de milliers de morts recensés.

- 2 juin 2015: le gouvernement expulse Toby Lanzer, coordinateur de l'aide humanitaire de l'ONu, après qu'il eut prédit «l'effondrement total» du pays.

- 19 juin 2015: selon un communiqué de l'Unicef, les forces armées ont perpétré des crimes atroces contre des enfants: émasculations, viols, gorges tranchées après qu'on les eut ligotés ensemble... Au moins 129 enfants ont été tués le mois précédent dans l'État d'Unité, dans le nord du pays, théâtre des combats parmi les plus violents du conflit.

\section{L'accord de paix}

— Juillet-août 2015: au cours des vingt mois de guerre, sept accords de cessez-le-feu ont été signés entre le gouvernement et l'armée rebelle, mais ils ont à chaque fois été vite rompus. En juillet 2015, sous la pression des ÉtatsUnis qui ont parrainé l'indépendance du Soudan du Sud, les dirigeants de l'Afrique de l'Est (Igad) se réunissent à Addis-Abeba et obligent les deux frères ennemis de Juba à négocier. Kiir et Machar signent le 26 août un accord de paix prévoyant le retour du leader rebelle dans la capitale et le retrait des forces étrangères, notamment de l'armée ougandaise qui combat depuis deux ans aux côtés des troupes gouvernementales sud-soudanaises.

- 13 octobre: l'Ouganda commence à retirer son contingent en application de l'accord de paix du 26 août.

- 24 décembre: dans une allocution télévisée, le président présente les vingt-huit gouverneurs des nouveaux États; ces nominations - effectuées en réaction à l'arrivée quelques jours auparavant à Juba d'une délégation 
de rebelles devant participer à la mise en œuvre des accords de paix et, notamment, à la formation d'un gouvernement d'union nationale sonnent comme une ultime provocation.

-7 janvier 2016 : le président Salva Kiir entérine la nomination de cinquante députés rebelles et forme un nouveau gouvernement de transition, avec des représentants des différentes factions de l'opposition.

- Avril: malgré l'accord signé par les deux parties, des combats sporadiques se produisent sur le terrain, jusqu'au retour de Riek Machar à Juba en avril 2016. L'accord d'Addis-Abeba prévoyait que l'essentiel des combattants des deux camps stationnerait désormais au-delà d'un rayon de 25 kilomètres autour de la capitale, Juba, à l'exception de 1400 soldats de la rébellion et de 3500 hommes de l'armée gouvernementale autorisés à y rester. À son retour à Juba, Riek Machar est intégré dans un gouvernement d'union nationale en tant que vice-président, poste qu'il avait occupé entre juillet 2011 et juillet 2013. Selon les dispositions de l'accord, ce gouvernement de transition, présidé par Salva Kiir, entre en fonction le 7 janvier 2016; il restera en place pendant trente mois.

\section{La recrudescence des violences}

- 8-11 juillet 2016 : les autorités sud-soudanaises avaient prévenu dès juin qu'en raison des tensions ethniques continuant de régner dans le pays, le cinquième anniversaire de son indépendance ne donnerait lieu à aucune célébration.

Une rumeur véhiculée par les médias sociaux, suivant laquelle Riek Machar aurait été pris en otage par les hommes du chef de l'État Salva Kiir, alors qu'il s'était rendu au palais présidentiel pour rencontrer celui-ci serait à l'origine des événements du 8 juillet. La capitale redevient ce jour-là le théâtre d'affrontements entre les forces du président et celles des rebelles aux ordres de son rival Riek Machar. Cette flambée de violence cause en quelques jours la mort 300 personnes et le déplacement de 42000 autres. Sous la pression de l'ONU, qui redoute une possible reprise des combats et leur extension à d'autres secteurs du pays, les belligérants signent un couvre-feu qu'ils imposent à leurs troupes.

- 13 juillet: quoique le cessez-le-feu décrété soit respecté, les pays étrangers continuent d'évacuer leurs ressortissants et les vols commerciaux restent suspendus.

- 17-18 juillet: suite à la récente flambée de violence à Juba, qui met gravement en péril l'accord de paix signé en août 2015 par les acteurs de la guerre civile, la crise sud-soudanaise est au cour des discussions $\mathrm{du} 27^{\mathrm{e}}$ sommet des chefs d'État de l'Union africaine (UA), les 17 et 18 juillet 2016 à Kigali. La présidente sortante de la commission de l'UA, 
la Sud-Africaine Nkosazana Dlamini-Zuma, affirme que «le continent ne peut pas rester les bras croisés face aux souffrances infligées aux enfants, femmes, hommes et jeunes gens du Soudan du Sud». Les chefs d'État africains réunis à Kigali doivent également décider de la fourniture de Casques bleus supplémentaires pour renforcer la mission de l'ONU au Soudan du Sud (opération Minuss). Il est enfin question de déployer une force régionale de protection des civils.

Les violents combats de Juba ont provoqué la fuite de Riek Machar et des forces du Spla-Io. La capitale se trouve désormais sous le contrôle des seules forces gouvernementales. Le 21 juillet, le président Salva Kiir somme Riek Machar de regagner la capitale sud-soudanaise sous 48 heures, faute de quoi il perdra son poste de vice-président. Mais ce dernier juge que sa sécurité ne peut plus être assurée à Juba et refuse. Le 25 juillet, Salva Kiir annonce alors la nomination d'un nouveau vice-président, le général Taban Deng Gai, de l'ethnie Nuer; cet ancien membre de la rébellion est pour sa part resté dans la capitale après les combats et se trouve être un rival de Riek Machar. Ce choix est condamné par le Mouvement populaire de libération du Soudan dans l'opposition (Splm-Io) qui rompt avec le nouveau vice-président.

Resté caché pendant plus d'un mois dans les environs de la capitale, Riek Machar quitte le Soudan du Sud à la mi-août et gagne la République Démocratique du Congo puis l'Éthiopie.

— En août 2016, un vote du Conseil de sécurité de l'ONU, qui passe outre le désaccord du gouvernement du Soudan du Sud, permet l'envoi d'un contingent de 4000 Casques bleus supplémentaires, principalement constitué de soldats éthiopiens, kényans et rwandais. Cette force, qui vient renforcer les 13500 Casques bleus déjà présents, devra se déployer à Juba pour sécuriser, notamment, l'aéroport et les bases de l'ONU.

— 17 août 2016: le Secrétaire général de l'ONU, Ban Ki-moon, décide de lancer une enquête spéciale indépendante pour déterminer les circonstances de l'attaque du 11 juillet contre l'hôtel Terrain à Juba, au cours de laquelle une personne a été tuée et plusieurs civils ont été violés et sauvagement battus par des hommes en uniforme.

— Le Conseil de sécurité des Nations unies, gravement préoccupé par les conflits en cours et par leurs conséquences humanitaires désastreuses sur la population du Soudan du Sud envoie, du 2 au 5 septembre, une mission à Juba. Il réagit également à la publication de rapports décrivant les violences sexuelles à grande échelle faites aux femmes et les massacres à caractère ethnique. Il appelle les parties à un cessez-le-feu immédiat. Le gouvernement sud-soudanais accepte finalement la force régionale de 4000 hommes destinée à sécuriser Juba. 
L'avenir dira si les deux leaders réussiront à apaiser les luttes de factions pour le pouvoir, qu'exacerbe la convoitise des richesses pétrolières du pays. (À cet égard, le groupe d'investigation The Sentry a dénoncé, le 13 septembre 2016, l'enrichissement personnel hors norme des chefs des belligérants.) Il témoignera de la capacité du gouvernement à respecter ses engagements et à reconstruire l'unité perdue depuis le référendum, unité sans laquelle ne saurait être construite la nation du Soudan du Sud.

L'évolution de la situation dépendra également de la position des acteurs internationaux, à commencer par les pays voisins dont les intérêts politiques et économiques divergent. L'Ouganda a ouvertement protégé en 2013 le régime en place en lui fournissant un appui militaire pour la sécurisation des zones pétrolières du Soudan du Sud. Riek Machar, lui, est soutenu par l'Éthiopie et le Soudan, lequel a intérêt à ce que son voisin du sud soit affaibli pour que s'émoussent les soutiens qu'il apporte aux différentes rébellions (Darfour, Kordofan du Sud et Nil Bleu) et que l'armée soudanaise puisse principalement se consacrer à la lutte contre ces rébellions.

Les intérêts divergents de ces pays regroupés au sein de l'Igad prédisposent mal cette organisation régionale à apaiser le conflit. De plus, la présence du pétrole au Soudan du Sud y crée des tensions entre grandes puissances, soucieuses d'y maintenir ou d'y développer leur zone d'influence.

\section{Une situation économique et humanitaire catastrophique}

Le conflit politico-ethnique ouvert depuis décembre 2013 a plongé le Soudan du Sud, qui se trouvait déjà en situation de fragilité, dans un profond marasme économique et y a provoqué une aggravation de la situation humanitaire. Malgré la conclusion d'un accord de paix en août 2015, le cessez-le-feu a été violé à de multiples reprises, ce qui a plongé le pays dans une insécurité croissante, notamment dans les États d'Unité, du Nil Supérieur, de Jonglei et de l'Équatoria occidental.

\section{Une crise économique profonde}

La survie de la majorité de la population sud-soudanaise dépend d'une agriculture de subsistance associant cultures et élevage. Il s'ensuit que les personnes déplacées perdent tout moyen de nourrir leur famille. Or, elles sont légion, d'où l'importance de l'aide humanitaire.

Au moment de l'indépendance, en juillet 2011, la situation économique n'était déjà pas brillante. Les royalties issues de la vente du pétrole ont, depuis, créé de la richesse, mais quasi uniquement dans la capitale. 
La population rurale n'a pas bénéficié de ces rentrées financières: sa vie quotidienne demeure inchangée, l'argent n'arrive pas dans les districts ruraux malgré les efforts de la communauté internationale. Cette inégalité est l'une des causes de l'exode rural massif en direction de la ville de Juba qui a grossi très rapidement.

Aux dires des dirigeants sud-soudanais, la situation de guerre avec le Soudan — qui a prévalu jusqu'en 2013 et qui a réduit les ressources de la jeune république — n'aurait pas aggravé la situation... puisque les populations rurales qui avaient survécu aux années de conflit n'avaient en rien bénéficié de la manne pétrolière...

L'économie du pays s'est fortement dégradée: la production de pétrole - dont, à son indépendance en 2011, le Soudan du Sud tirait $98 \%$ de ses recettes - a diminué de plus de la moitié, pour s'établir à moins de 150000 barils par jour (au lieu de 380000 ), et l'inflation s'est envolée pour atteindre les $600 \%$ sur un an; la monnaie nationale s'est, par la suite, considérablement dépréciée: si le taux officiel est de trente livres sud soudanaises pour un dollar, le taux réel (en septembre 2016) était de soixante-dix livres pour un dollar, d'où une hausse spectaculaire du prix des denrées de première nécessité qui, pour l'essentiel, sont importées.

Autres manifestations du marasme économique, en plus de la flambée des prix alimentaires, des prix de l'énergie et du coût de la vie: l'effondrement du commerce et des marchés locaux, ainsi que l'épuisement des réserves alimentaires. Selon les chiffres du Programme alimentaire mondial (Pam), sur les marchés de Juba, en août 2016, les légumes se vendaient entre 45 et $80 \%$ plus cher que d'habitude et le prix du fuel avait, lui, augmenté de 70 à $80 \%$.

Les activités commerciales pâtissent de l'insécurité et de l'inflation des frais de transport qu'elle induit. Il est difficile de trouver des denrées essentielles. Le système bancaire fonctionne difficilement.

Le budget 2016-2017, adopté le 27 août 2016 (trois fois supérieur au budget précédent, suite à la mise en œuvre des accords de paix), se monte à environ un milliard de dollars... sans que l'on connaisse la provenance des recettes correspondantes.

L'assistance humanitaire absorbe l'essentiel de l'aide extérieure, alors qu'elle était vouée, avant le début de la guerre civile, à de nombreux projets de développement en cours ou programmés.

\section{Une crise humanitaire dramatique}

Plus de 2,6 millions de Soudanais du Sud (un sur cinq) ont été contraints de quitter leurs villages pour fuir les violences, dont 1,6 million à l'intérieur du pays et plus de 900 ooo dans les pays voisins, surtout en Éthiopie, 
au Soudan, au Kenya et en Ouganda, où, selon le Haut-Commissariat des Nations unies pour les réfugiés (HCR), dans les semaines qui ont suivi les combats de juillet 2016 à Juba, plus de 75000 personnes en grande détresse se sont réfugiées.

L'insécurité alimentaire pousse à présent la population dans ses derniers retranchements. Une personne sur trois se trouve dans une situation de grande insécurité alimentaire. On estime à plus de 686000 le nombre d'enfants de moins de cinq ans (soit un sur quatre) souffrant de malnutrition aiguë sévère. D’après le dernier rapport du Pam, le pays est à son plus haut niveau d'insécurité alimentaire depuis le début du conflit en 2013. Or, cette crise alimentaire, l'une des pires au monde, risque de s'aggraver encore si la situation politique demeure instable.

La situation sanitaire est elle aussi dramatique: $87 \%$ de la population n'ont pas accès à des installations sanitaires améliorées; seulement $47 \%$ ont accès à de l'eau potable. Plusieurs centaines de cas de choléra ont été enregistrés dans le pays depuis le mois de juillet 2016 : cette maladie est endémique et les recrudescences sont observées en saison des pluies; les déplacements de population favorisent son extension géographique. La situation des femmes victimes de violences sexuelles et des 15000 à 16 ooo enfants actuellement enrôlés dans les différentes factions armées en présence est alarmante. Un chiffre résume le drame actuel: à la fin 2016 plus de six millions de Soudanais du Sud dépendaient de l'aide humanitaire pour leur survie.

\section{L'aide humanitaire dans un contexte difficile}

Les travailleurs humanitaires sont soumis à des violences, à des harcèlements et à des intimidations qui ont déjà provoqué le décès de cinquantecinq d'entre eux. De plus, les organisations humanitaires doivent souvent faire face à la saisie de leurs actifs, aux obstructions de l'administration, à des taxations abusives et à des détournements illégaux de l'aide d'urgence: selon le rapport de l'European Commission Humanitarian Office (Есно, juillet 2016), toutes les forces armées se sont livrées à des pillages systématiques de l'aide d'urgence à la faveur des récents combats.

II faudrait que s'installe une véritable transition vers la démocratie, que s'instaure une réelle transparence dans l'utilisation des ressources pétrolières, que les efforts de développement touchent aussi les zones rurales dont les populations n'ont connu que la guerre, les répressions, les famines... L'avenir nous dira si le plus jeune État du monde réussira à relever ces défis et si ses forces vives pourront enclencheront un développement soutenable au profit de tous, non de quelques-uns 